\title{
An Application of the Curvature Scale Space Shape Descriptor for Forensic Human Identification Based on Orthopantomograms
}

\author{
Dariusz Frejlichowski and Piotr Czapiewski \\ West Pomeranian University of Technology, Szczecin, \\ Faculty of Computer Science and Information Technology, \\ Zolnierska 52, 71-210, Szczecin, Poland \\ \{dfrejlichowski,pczapiewski\}@wi.zut.edu.pl
}

\begin{abstract}
This paper is devoted to the problem of forensic human identification based on the application of dental biometrics. For this purpose digital orthopantomogram images are used. The complete scheme of the approach is described. It starts with the image enhancement by means of the Laplacian pyramid decomposition. Secondly, the segmentation of an image into sub-images covering single teeth is performed. The process is based on determining the line between the upper and lower jaws by means of integral projections and, later, image intensity and types of teeth. For each obtained sub-image, covering a single tooth, its shape is extracted and the outer contour of this shape is traced. Thus, a shape descriptor can be applied in order to represent an object for the later identification. In this paper, the Curvature Scale Space algorithm is applied and investigated as one of the elements of the biometric identification approach based on digital orthopantomogram images.
\end{abstract}

Keywords: dental images, forensics, shape descriptors, Curvature Scale Space.

\section{Introduction}

Dental records are widely used in forensic human identification as one of the most efficient biometric modalities in this application [1]. They are often used in the process of establishing the identity of a person, together with DNA and fingerprints. The popularity of dental biometrics in judicial proceedings comes from their property of discrimination and the robustness to decomposition 2], where for example automatic face recognition would fail [3, 4]. It has led to several attempts for the development of an identification system, such as WinID that compares dental records previously codified by an expert. The second example, which is closer to the approach assumed in the works described in this paper, is the Automated Dental Identification System (ADIS). One of the stages in this system is the automatic extraction of dental biometrics [5]. Whereas WinID applies dental works as a basis for identification, ADIS utilizes teeth shapes extracted from radiograms for this purpose. The ADIS system is an important tool 
used in the work of the Dental Task Force (DTF), a special service created by the Federal Bureau of Investigation (FBI) [6].

The analysis of radiographic images is extremely significant for various medical and forensic purposes, and hence many general methods related to radiograms have been described [7-9]. However, the specifics of the dental image, and especially of orthopantomograms, must be taken into consideration in order to properly discern between teeth and represent their shape.

The automatic system for the identification of persons based on digital orthopantomograms is composed of image enhancement and segmentation, and feature extraction and recognition. In the first step one can apply, for example, the wavelet-transform [10, 11] or Laplacian pyramid [12, 13]. During the segmentation process the integral projections [15, 16] or active contour models [12] have been applied so far. The extraction of particular teeth shapes is performed using active shapes [14], line scanning [15] or watersheds [17]. The first two algorithms were developed specifically for the intraoral images. Hence, they fail when applied in pantomograms. This problem results from frequent occlusions. The third mentioned method performs better in this case. In the last stage any of the shape description and recognition approaches can be applied.

In this paper the whole algorithm is described. Firstly, the methods for image enhancement are presented. Next, the segmentation and localisation of the object of interest - single teeth — is provided. Both methods are discussed in the second section. Later, in the third section, the algorithm for the representation of the extracted contours is described. The fourth section discusses the experimental conditions and results. Finally, the last section concludes the paper.

\section{Image Enhancement, Segmentation and the Extraction of Teeth Shapes}

The image enhancement applied for the pantomograms starts with the Laplacian decomposition, proposed in [18. The Laplacian pyramid's layers are derived through the subtraction of consecutive layers of a Gaussian pyramid. This process is represented by means of the following formula:

$$
X_{k}=\downarrow\left(\bar{X}_{k-1}\right), \quad L_{k}=X_{k-1}-\uparrow\left(X_{k}\right),
$$

where $X_{0}$ is the original image, $\downarrow(X)$ and $\uparrow(X)$ represent the process of downsampling and upsampling the image by a factor of $2, \bar{X}_{k}$ is a low-pass filtered image of $X_{k}$, and $L_{k}$ is the successive layer of the Laplacian pyramid. The lowpass filtering is performed using the Gaussian filter.

For the Laplacian pyramid, a contrast equalisation function is used (as described in [19]):

$$
f(x)=a\left(\frac{x}{|x|}\right)|x|^{p}
$$

where " $x$ is normalized to the range $[-1,1]$ and the factor $a$ is needed for rescaling the resulting image to the original dynamic range" [19]. The exponent $p$ controls 
the degree of non-linearity. According to further modifications provided by [20], a new representation of the Laplacian pyramid, applied in our approach, can be formulated as:

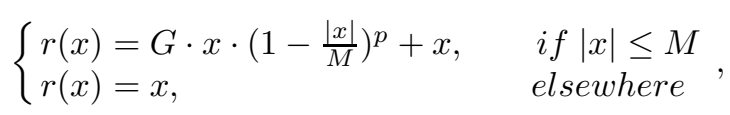

where $M$ is the upper limit for linear enhancement and $G$ is the constant gain.

In our previous experiments [13, 16, 17] the best results were obtained when using the following combination of methods: 1) the averaging of the two Laplacian pyramid layers next to last, 2) unsharp filter on the second layer, 3) contrast enhancement, based on the eq.22. This approach was used at the stage of image pre-processing.

The second stage consists of the segmentation of an image. Firstly, the upper and lower jaws are separated by means of integral projections. Usually, the initial starting point for this process is established by the user. However, our goal is to fully automate this process. Hence, in the method described in this paper, this point is selected as the horizontal integral projection around the centre of the image with the lowest value. Taking into account the characteristics of a pantomogram (the separating line is not horizontal), only some of the pixels $(20 \%$ of the image width), which lay closest to the frontal teeth gap selected earlier, are used for the derivation of projections. The area between the necks of two adjacent teeth is easy to locate on the pantomogram. Thus, the aforementioned line is applied for the location of the position of the neck for each tooth. Later, points lying on splines representing gaps between the necks of two adjacent teeth are selected. For the upper and lower jaw, an array of values is obtained, comprised of the intensity values of the points lying on the splines going through their respective dental pulps. The sharp spikes visible on the plot representing these values correspond to the dark regions surrounded by light intensities, what indicates a gap between teeth necks. For the matching process only small subsets (with the pre-assumed number of elements) of the values in the array are selected.

For the indication of a spike value corresponding to the gap between the necks of teeth for a processed tooth, lying on the position $p_{c}$, the Bayesian probability $P\left(x_{i}, p_{c}\right)$ calculated for each point on the curve $\left(x_{i}\right)$ is used:

$$
P\left(x_{i}, p_{c}\right)=I\left(x_{i}\right) G\left(x_{i}, p_{c}\right) D\left(x_{i}, p_{c}\right),
$$

where:

$I$ - the intensity of the range filtered and inverted original image in the point $x_{i}$,

$G$ - a discrete Gaussian function with expected value equal to the horizontal position of the last detected gap, displaced left or right (depending on the current search direction) by the amount of pixels that is equal to the average width of the tooth at position $p_{c}$, 
$D$ - a function introduced to limit the number of pixels analysed in each iteration. It is equal to 1 for points, for which the horizontal distance from the previous detected gap is between $75 \%$ and $175 \%$ of the expected width of a tooth on position $p_{c}$, with regard to the current search direction, and equal to 0 elsewhere.

At each iteration, the current maximal value obtained using the above formula is added to the list of gap positions and it is used as a starting point for the next iteration. The iterations end at the border of an image or when the number of gaps for either the upper or lower jaw is equal to 8. Usually, the obtained gap locations are reliable indicators for the space between the teeth. However, sometimes the vertical line is insufficient for the distinction between two occluding teeth. Thus, additionally another point between them is localised by means of a greedy algorithm, that iteratively moves one pixel towards the radiogram's top or bottom, selects the pixel in horizontal neighbourhood with the highest intensity on the inverted, range-filtered image and uses it as the basis for the next iteration. The number of iterations is equal to the half length of an average tooth on a pantomogram. The last obtained point is taken as the second point applied for the separation. Using it, as well as the previously derived point, the segmentation line is established.

Later, the areas lying below the teeth roots are removed by means of the curve separating jaws. This line is shifted vertically until such an alignment is obtained, where the sum of pixels through which it passes is smaller than the surrounding result. That indicates the area between the teeth line and the cheekbone.

An exemplary pantomogram enhanced and segmented by means of the described approach is provided in Fig. 1.

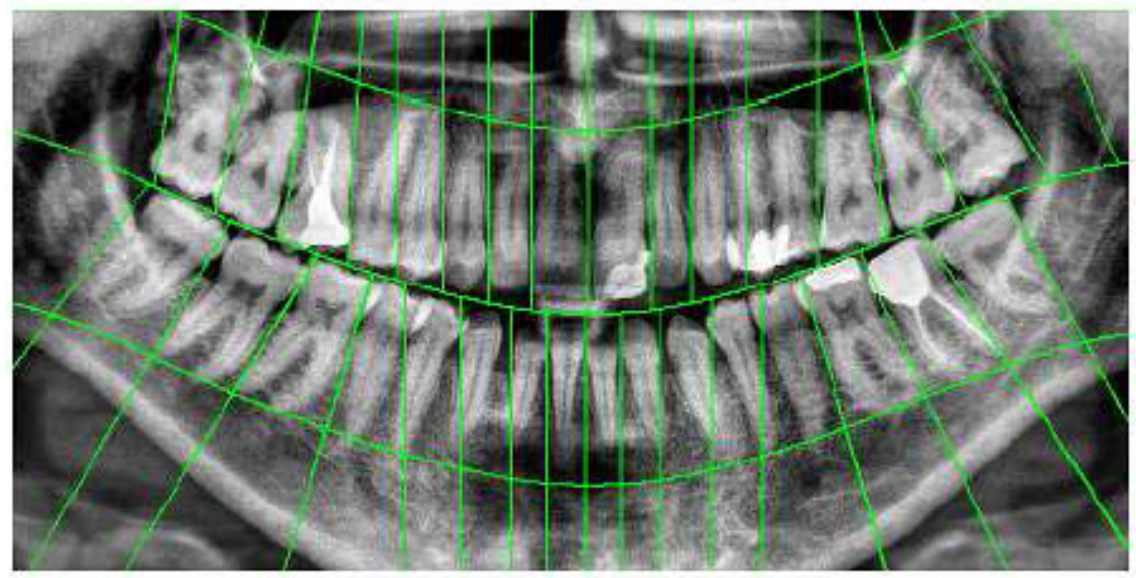

Fig. 1. An exemplary result of the image pre-processing and segmentation, described in this section 
After the segmentation of the image, we can extract the shapes of particular teeth. Firstly, in order to lower the influence of noise and enlarge the areas with similar intensity, the opening operation on the image is performed. Later, the entropy filtering is applied. It detects edges of areas with similar colours. Then, the watershed algorithm is used in order to divide the image into small segments. The dimensions of the obtained areas depends on the size of a structuring element used in the morphological opening - the larger the element, the larger size of the segments.

For each segment the following features are obtained: the centroid, the normalised mean intensity, and the normalised vertical distance from the centroid to the curve separating the jaws. Next, the distance between obtained centroids is derived, and 50 of the closest segments to a processed segment are selected for the calculation of the average intensity. The distinction value of the $i$-th segment is derived using the equation:

$$
D(i)=\sum_{j=1}^{N} \max (\bar{I}(i)-\bar{I}(j), 0),
$$

where: $\bar{I}(i)$ and $\bar{I}(j)$ are the average intensities of $i$-th and $j$-th segments. These values help in determining whether a segment is brighter than its surrounding segments or not.

Later, a fitness function is calculated in order to select the segments belonging to the tooth. The values of this function depend on the type of the tooth. For the incissors only the distinction function and vertical distance from the jaws separating curve are used, with the weights of 0.7 and 0.3 , respectively. For other types of teeth the average intensity is also included, with the weights of 0.4 (distinction function), 0.4 (average intensity) and 0.2 (vertical distance from the jaws separating curve).

When all fitness functions for segments are obtained, the process of selection of those belonging to a tooth begins. Basing on the performed experiments we could assume that a fitness function value has to be larger than 0.4 for incissors, and 0.5 for the canine and first premolar. For other types of teeth the threshold is established as the average intensity multiplied by 0.8 . The selected regions are morphologically dilated, what results in the removal of borders that separate them. Finally, the contour of a tooth can be traced and extracted (see Fig. 2).
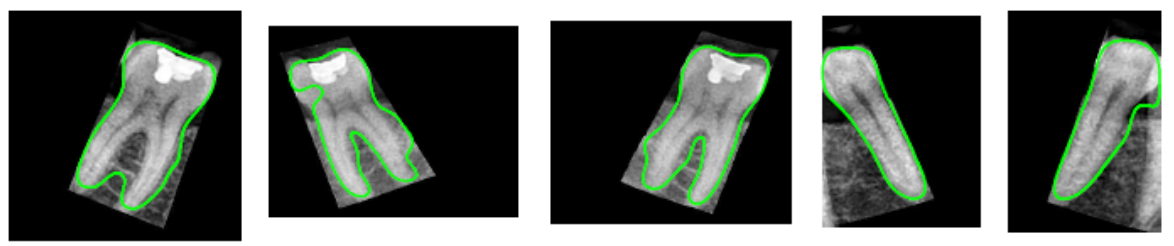

Fig. 2. Examples of the segmented teeth images 


\section{Representation of the Teeth Contours Using the Curvature Scale Space Transform}

The extracted contours of teeth are described using the Curvature Scale Space (CSS) transform [21, 22]. The CSS representation is calculated using the convolution of a path-based parametric representation of a curve extracted from the boundary of processed shape with a Gaussian function of increasing variance $\sigma^{2}$. The zeros of curvatures of the convolved curves are extracted and combined in a scale space representation. Those values are derived during the evolution of the planar curve changed by the expanding Gaussian function.

The closed planar curve $r$ is represented parametrically for axes $x$ and $y$ using the normalized arc length parameter $u$ :

$$
r(u)=\{x(u), y(u) \mid u \in[0,1]\} .
$$

The evolved curve is represented as $\Gamma_{\sigma}$ :

$$
\Gamma_{\sigma}(u)=\{\chi(u, \sigma), \psi(u, \sigma)\},
$$

where:

$$
\chi(u, \sigma)=x(u) \otimes g(u, \sigma), \quad \psi(u, \sigma)=y(u) \otimes g(u, \sigma)
$$

and:

$\otimes$ - the convolution operator,

$g$ - Gaussian function of width $\sigma$, calculated using the formula:

$$
g(u, \sigma)=\frac{1}{\sigma \sqrt{2 \pi}} e^{-u^{2} / 2 \sigma^{2}} .
$$

The curvature $\kappa$ of $\Gamma$ can be formulated as:

$$
\kappa(u, \sigma)=\frac{\chi_{u}(u, \sigma)-\psi_{u u}(u, \sigma)-\chi_{u u}(u, \sigma)-\psi_{u}(u, \sigma)}{\left(\chi_{u}(u, \sigma)^{2}+\psi_{u}(u, \sigma)^{2}\right)^{3 / 2}},
$$

where:

$$
\begin{aligned}
& \chi_{u}(u, \sigma)= \frac{\partial}{\partial u}(x(u) \otimes g(u, \sigma))=x(u) \otimes g_{u}(u, \sigma), \\
& \chi_{u u}(u, \sigma)= \frac{\partial^{2}}{\partial^{2} u}(x(u) \otimes g(u, \sigma))=x(u) \otimes g_{u u}(u, \sigma), \\
& \psi_{u}(u, \sigma)=y(u) \otimes g_{u}(u, \sigma), \\
& \psi_{u u}(u, \sigma)=y(u) \otimes g_{u u}(u, \sigma) .
\end{aligned}
$$

Finally, the CSS image $I_{c}$ gives in result a multi-scale representation of zero crossing points:

$$
I_{c}=\{(u, \sigma) \mid \kappa(u, \sigma)=0, u \in[0,1], \sigma \geq 0\} .
$$

The last stage of the descriptor's construction is the selection of contour maxima locations in CSS image $I_{c}$. They are used as a representation for a shape. 
The similarity between two represented objects is calculated through matching between two sets of maxima, representing them. The method firstly finds any possible changes in orientation in one of the two shapes, then a circular shift is applied in order to compensate their influence. Later the Euclidean distance is calculated between the two sets, indicating the dissimilarity measure.

\section{Discussion on the Experimental Conditions and Obtained Results}

The approach described in the previous two sections was experimentally investigated using 60 digital pantomograms, belonging to 30 individuals. For each person two images were applied that were taken at various time points. One image was treated as a template pattern and the second as a test object.

After the pre-processing and segmentation of an image and the extraction of teeth shapes, each obtained contour was represented using the Curvature Scale Space transform. As can be seen on Fig. 3, the CSS shape descriptors obtained for different teeth differ significantly, which confirms the validity of their application in the discussed problem. For the derivation of the similarity between two descriptions the method discussed in the previous section was applied.
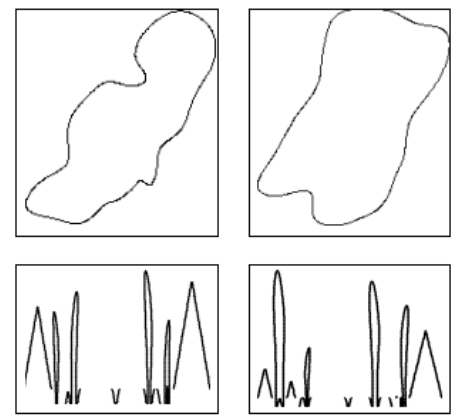
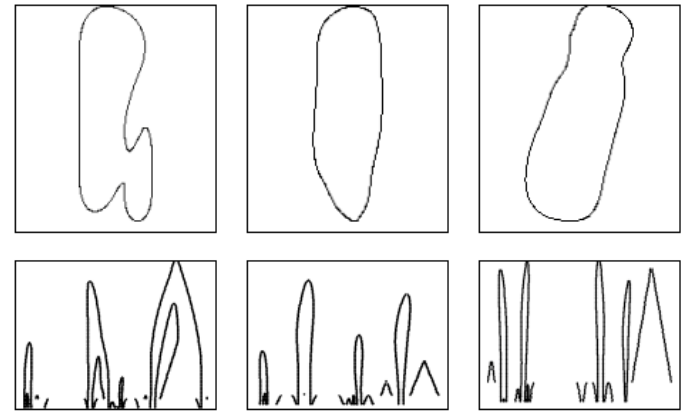

Fig. 3. Examples of teeth contours and obtained CSS descriptors

For further processes the location of each tooth was required, because the matching was performed on the level of particular tooth types - incisors extracted from pantomograms under recognition were matched with incisors from all base images, canines were compared with canines, and so on. The best recognition results were achieved when using the molars. Nevertheless, other tooth types should also be taken into account, since for certain people this type of tooth was not always present.

The indication process was performed separately for each of the four quarters of a pantomogram image. For each of them, the comparison started from the back of the jaw and continued towards the center of the image. If a tooth was present on a particular position in both the test and template sub-images, matching 
would be performed. The Euclidean distance indicated the template person for a single tooth. The template class with the largest number of indications was selected as the recognized person.

Using the described approach an experiment was performed. As a result of this experiment 20 of the 30 test objects were identified properly, which gives a recognition rate equal to $67 \%$. Although this result may seem far from ideal it is mainly connected with the difficult character of the digital orthopantomogram images.

\section{Conclusions}

An approach for automatic people identification using pantomogram images was described in this paper. It consisted of four stages: image enhancement, image segmentation, tooth localisation, and recognition by means of tooth shapes for a particular person. The shapes were represented using the Curvature Scale Space transform and matched with the shapes stored in the template base. The largest number of correctly indicated teeth decided about the selection of the template class that was most similar to the test image.

During the experiment 60 pantomogram images acquired from 30 people were used - half of the images were selected as templates assigned to particular persons, and the other half was used as test objects. This means that two images obtained at various points in time were used for each person. The achieved identification rate was equal to $67 \%$, what is not enough but nonetheless one has to keep in mind the particularly difficult character of the dental orthopantomogram images. Nevertheless, future works on this problem are planned. First, other shape representation techniques can be applied and experimentally inves-

tigated, including evaluation of potential hardware implementation [23]. Second, other methods for the pre-processing of the image can be analysed in order to emphasise the locations of particular teeth before the extraction of their shapes. Finally, other methods for making a final decision about the identified person could be developed and evaluated.

Acknowledgements. The authors of this paper wish to thank gratefully to R. Wanat, MSc for his significant help in developing and investigating the described approach.

\section{References}

1. Bowers, M.C.: Forensic Dental Evidence. Elsevier Academic Press (2004)

2. Lee, S.S., Choi, J.H., Yoon, C.L., Kim, C.Y., Shin, K.J.: The Diversity of Dental Patterns in Orthopantomography and its Significance in Human Identification. Journal of Forensic Science 49(4), 784-786 (2004)

3. Spaun, N.A.: Face Recognition in Forensic Science. In: Li, S.Z., Jain, A.K. (eds.) Handbook of Face Recognition, pp. 655-670. Springer (2011)

4. Shchegoleva, N.: Facial Surface Reconstruction in 3D Format. Journal of Theoretical and Applied Computer Science 6(4), 37-50 (2012) 
5. Fahmy, G., et al.: Towards an Automated Dental Identification System (ADIS). In: Zhang, D., Jain, A.K. (eds.) ICBA 2004. LNCS, vol. 3072, pp. 789-796. Springer, Heidelberg (2004)

6. Nassar, D., Ammar, H.H.: A Prototype Automated Dental Identification System (ADIS). In: Proc. of the 2003 Annual National Conference on Digital Government Research, pp. 1-4. Digital Government Society of North America (2003)

7. Gut, P., Chmielewski, L., Kukolowicz, P., Dabrowski, A.: Edge-Based Robust Image Registration for Incomplete and Partly Erroneous Data. In: Skarbek, W. (ed.) CAIP 2001. LNCS, vol. 2124, pp. 309-316. Springer, Heidelberg (2001)

8. Bator, M., Chmielewski, L.J.: Finding Regions of Interest for Cancerous Masses Enhanced by Elimination of Linear Structures and Considerations on Detection Correctness Measures in Mammography. Pattern Analysis and Applications 12(4), 377-390 (2009)

9. Kukolowicz, P.F., Dabrowski, A., Gut, P., Chmielewski, L., Wieczorek, A., Kedzierawski, P.: Evaluation of Set-up Deviations During the Irradiation of Patients Suffering from Breast Cancer Treated With Two Different Techniques. Radiotherapy and Oncology 75(1), 22-27 (2005)

10. Lu, J., Healy Jr., D.M.: Contrast Enhancement of Medical Images Using Multiscale Edge Representation. Optical Engineering 33(7), 2151-2161 (1994)

11. Dippel, S., Stahl, M., Wiemker, R., Blaffert, T.: Multiscale Contrast Ehnahncement for Radiographies: Laplacian Pyramid Versus Fast Wavelet Transform. IEEE Trans. on Medical Imaging 21(4), 343-353 (2002)

12. Zhou, J., Abdel-Mottaleb, M.: A Content-Based System for Human Identification Based on Bitewing Dental X-ray Images. Pattern Recognition 38(11), 2132-2142 (2005)

13. Frejlichowski, D., Wanat, R.: Application of the Laplacian Pyramid Decomposition to the Enhancement of Digital Dental Radiographic Images for the Automatic Person Identification. In: Campilho, A., Kamel, M. (eds.) ICIAR 2010, Part II. LNCS, vol. 6112, pp. 151-160. Springer, Heidelberg (2010)

14. Chen, H., Jain, A.K.: Automatic Forensic Dental Identification. In: Jain, A.K., Flynn, P., Ross, A.A. (eds.) Handbook of Biometrics, pp. 231-251. Springer (2008)

15. Jain, A.K., Chen, H.: Matching of Dental X-ray Images for Human Identification. Pattern Recognition 37(7), 1519-1532 (2004)

16. Frejlichowski, D., Wanat, R.: Automatic Segmentation of Digital Orthopantomograms for Forensic Human Identification. In: Maino, G., Foresti, G.L. (eds.) ICIAP 2011, Part II. LNCS, vol. 6979, pp. 294-302. Springer, Heidelberg (2011)

17. Frejlichowski, D., Wanat, R.: Extraction of Teeth Shapes from Orthopantomograms for Forensic Human Identification. In: Real, P., Diaz-Pernil, D., MolinaAbril, H., Berciano, A., Kropatsch, W. (eds.) CAIP 2011, Part II. LNCS, vol. 6855, pp. 65-72. Springer, Heidelberg (2011)

18. Burt, P.J., Adelson, E.H.: The Laplacian Pyramid as a Compact Image Code. IEEE Trans. on Communications 31(4), 532-540 (1983)

19. Vuylsteke, P., Schoeters, E.: Image Processing in Computed Radiography. In: Proc. of International Symposium on Computerized Tomography for Industrial Applications and Image Processing in Radiology, pp. 87-101. DGZfP (1999)

20. Stahl, M., Aach, T., Buzug, T.M., Dippel, S., Neitzel, U.: Noise-Resistant WeakStructure Enhancement for Digital Radiography. In: Hanson, K.M. (ed.) Medical Imaging 1999: Image Processing. SPIE, vol. 3661, pp. 1406-1417. SPIE (1999) 
21. Mokhtarian, F.: Silhouette-Based Occluded Object Recognition Through Curvature Scale Space. Machine Vision and Applications 10(3), 87-97 (1997)

22. Roh, M.-C., Christmas, B., Kittler, J., Lee, S.-W.: Robust Player Gesture Spotting and Recognition in Low-Resolution Sports Video. In: Leonardis, A., Bischof, H., Pinz, A. (eds.) ECCV 2006. LNCS, vol. 3954, pp. 347-358. Springer, Heidelberg (2006)

23. Frejlichowski, D.: Analysis of Possible System-Level Hardware Implementation of the Selected Shape Description Algorithms. Journal of Theoretical and Applied Computer Science 6(4), 51-58 (2012) 